\title{
Establishing a South African national framework for COVID-19 surgical prioritisation
}

\author{
K M Chu, ${ }^{1}$ MD, MPH, FACS, FASCRS; E O Owolabi, ${ }^{1}$ BNSc, MCur, PhD; \\ M Smith, ${ }^{2}$ MB BCh, FCS (SA), FEBS (HPB), RCSEd (Fellowship ad hominem); T C Hardcastle, ${ }^{3}$ MB ChB, MMed (Chir), FCS (SA), PhD; \\ S Maswime, ${ }^{4}$ MB ChB, FCOG (SA), MMed (Obstet Gynaecol), PhD; H Geduld, ${ }^{5}$ MB ChB, Dip PEC, MMed (Emerg Med), FCEM; \\ P D Gopalan, ${ }^{6} \mathrm{MB}$ ChB, FCA (SA), Crit Care (HPCSA), PhD; J Marco, ${ }^{1}$ BA, MA; M Mendelson, ${ }^{7} \mathrm{MBBS}, \mathrm{PhD}$; \\ B M Biccard, ${ }^{8}$ MB ChB, FFSRCSI, FCA (SA), MMedSci, PhD; L Cairncross, ${ }^{4}$ MB ChB, FCS (SA), MMed (Surg); \\ on behalf of the South African National Surgical Obstetric Anaesthesia Plan Task Team
}

\author{
${ }^{1}$ Centre for Global Surgery, Department of Global Health, Faculty of Medicine and Health Sciences, Stellenbosch University, Cape Town, \\ South Africa \\ ${ }^{2}$ Department of Surgery, Faculty of Health Sciences, University of the Witwatersrand and Chris Hani Baragwanath Academic Hospital, \\ Johannesburg, South Africa \\ ${ }^{3}$ Department of Surgery, School of Clinical Medicine, University of KwaZulu-Natal and Inkosi Albert Luthuli Central Hospital, Durban, \\ South Africa \\ ${ }^{4}$ Global Surgery Division, Department of Surgery, Faculty of Health Sciences, University of Cape Town, South Africa \\ ${ }^{5}$ Division of Emergency Medicine, Department of Family and Emergency Medicine, Faculty of Medicine and Health Sciences, Stellenbosch \\ University, Cape Town, South Africa \\ ${ }^{6}$ Discipline of Anaesthesiology and Critical Care, School of Clinical Medicine, University of KwaZulu-Natal, Durban, South Africa \\ ${ }^{7}$ Division of Infectious Diseases and HIV Medicine, Groote Schuur Hospital and Faculty of Health Sciences, University of Cape Town, South Africa \\ ${ }^{8}$ Department of Anaesthesia and Perioperative Medicine, Groote Schuur Hospital and Faculty of Health Sciences, University of Cape Town, \\ South Africa
}

Corresponding author: KM Chu (kchu@sun.ac.za)

\begin{abstract}
Background. Since the start of the COVID-19 pandemic, surgical operations have been drastically reduced in South Africa (SA). Guidelines on surgical prioritisation during COVID-19 have been published, but are specific to high-income countries. There is a pressing need for context-specific guidelines and a validated tool for prioritising surgical cases during the COVID-19 pandemic. In March 2020, the South African National Surgical Obstetric Anaesthesia Plan Task Team was asked by the National Department of Health to establish a national framework for COVID-19 surgical prioritisation.

Objectives. To develop a national framework for COVID-19 surgical prioritisation, including a set of recommendations and a risk calculator for operative care.

Methods. The surgical prioritisation framework was developed in three stages: (i) a literature review of international, national and local recommendations on COVID-19 and surgical care was conducted; (ii) a set of recommendations was drawn up based on the available literature and through consensus of the COVID-19 Task Team; and (iii) a COVID-19 surgical risk calculator was developed and evaluated. Results. A total of 30 documents were identified from which recommendations around prioritisation of surgical care were used to draw up six recommendations for preoperative COVID-19 screening and testing as well as the use of appropriate personal protective equipment. Ninety-nine perioperative practitioners from eight SA provinces evaluated the COVID-19 surgical risk calculator, which had high acceptability and a high level of concordance (81\%) with current clinical practice.

Conclusions. This national framework on COVID-19 surgical prioritisation can help hospital teams make ethical, equitable and personalised decisions whether to proceed with or delay surgical operations during this unprecedented epidemic.
\end{abstract}

S Afr Med J. Published online 23 March 2021. https://doi.org/10.7196/SAMJ.2021.v111i5.15603

Since the beginning of 2020, the COVID-19 pandemic has swept across the globe at an unprecedented pace. As of 1 February 2021, there were $>102$ million cases in $>188$ countries. ${ }^{[1]}$ The COVID19 pandemic brought about significant changes in health-seeking behaviour and healthcare service delivery, such as decreased inpatient admissions and outpatient visits in order to conserve hospital resources for the large number of persons with COVID-19. ${ }^{[2]}$ In March 2020, non-essential surgical care was greatly reduced in some countries, with an estimated 28 million procedures deferred during the first 12 weeks of the pandemic. ${ }^{[3-7]}$ Hospitals only performed essential surgery, including trauma-related life-saving procedures, and urgent planned surgery (such as for cancer), resulting in countries experiencing huge surgical backlogs ${ }^{[8-13]}$ that will have significant long-term effects. Pre-COVID-19, surgical health systems in sub-Saharan Africa were already limited and inequitable, with long operation waiting times in many countries. ${ }^{[14]}$ The pandemic has exacerbated this lack of access to surgical care.

COVID-19 was first described in South Africa (SA) in March 2020, but despite severe measures such as a national lockdown, which after a year is still ongoing, the country has had over one million confirmed infections, representing more than half of all the cases on the continent. ${ }^{[1]}$ While the first wave peaked in July 2020 and the second wave in early 2021, the pandemic will not be controlled until vaccines are widely available and national herd immunity is reached. 
As in most countries worldwide, SA hospitals reduced healthcare services by limiting outpatient clinics and non-essential surgeries. ${ }^{[8]}$ According to one estimate, procedure cancellations in SA in the first 12 weeks of the pandemic created a surgical backlog of $>150000$ cases, which it is expected will take almost 2 years to catch up if healthcare facilities are able to manage a $10 \%$ increase in surgical capacity post-COVID-19. ${ }^{[4,15]}$ Since the pandemic has gone on for almost a year, this backlog is expected to be even greater now.

As SA public and private hospitals consider which operations to perform while balancing local pandemic factors and resources, there is a need for a national guideline around prioritisation of surgical care to balance access, equity, urgency, and COVID-19 infection risks to patients and staff. Currently, some hospitals have their own prioritisation systems, sometimes with operative theatre selection committees, while others allow their individual surgeons to decide on whom to operate. ${ }^{[8]}$

Guidelines on surgical prioritisation during COVID-19 have been published, but are specific to high-income countries. ${ }^{[1,17]}$ These guidelines recommend certain prerequisites such as decreasing local daily COVID-19 cases, adequate personal protective equipment (PPE) for surgical staff, and availability of preoperative SARS-CoV-2 polymerase chain reaction (PCR) testing. In addition, a US surgical group created the medically necessary, time-sensitive (MeNTs) scoring system to help with decision-making on specific surgical patients during COVID-19, considering patient, disease, procedural and local pandemic factors. ${ }^{[18]}$ In low- and middle-income countries, return to 'normal' may be challenging given the lack of PPE, limited preoperative SARS-CoV-2 PCR testing, and fewer intensive care unit and ward beds in these lower-resourced settings. There is a pressing need for context-specific guidelines and a validated tool for prioritising surgical cases during the COVID-19 pandemic. Return to pre-pandemic volumes of surgery may not be possible for many months or for years, depending on local pandemic factors.

In early March 2020, the SA National Department of Health $(\mathrm{NDoH})$ convened a task team to create a National Surgical Obstetric and Anaesthesia Plan. This group consists of academic surgeons, anaesthesiologists, obstetrician/gynaecologists, emergency medicine physicians and critical care specialists from five SA universities and the NDoH. A COVID-19 subgroup was formed to establish an SA national framework for COVID-19 surgical prioritisation.

\section{Objectives}

The primary objective of this publication was to describe the development of this national framework, including a set of recommendations and a COVID-19 risk calculator for operative care.

\section{Methods}

The SA national framework for COVID-19 surgical prioritisation was developed in three stages: (i) a literature review of international, national and local recommendations on COVID-19 and surgical care was conducted; (ii) a set of recommendations was drawn up based on the available literature and through consensus of the COVID-19 Task Team; and (iii) a COVID-19 surgical risk calculator was developed and evaluated.

\section{Literature review}

MEDLINE was searched via PubMed and Google Scholar for the key words 'surgery', 'COVID-19', 'operation' and 'prioritisation'. All publications including commentaries and research articles from any country were included through 15 June 2020. In addition, grey literature, or in-house policy documents, from SA hospitals on COVID-19 surgical prioritisation were included. Each publication was reviewed by a member of the COVID-19 Task Team and key points were extracted.

\section{National recommendations and COVID-19 risk calculator}

Based on the literature review and discussions with the COVID19 Surgical Task Team members who work in various hospitals nationwide, national recommendations and a COVID-19 risk calculator were created through consensus.

\section{Evaluation of recommendations and COVID-19 risk calculator \\ Study design}

A cross-sectional study conducted via an online survey between 11 September and 15 November 2020 was used to evaluate the recommendations and COVID-19 surgical risk calculator. Participants were recruited through professional societies, hospital and academic department meetings, and snowball sampling. Surgeons, anaesthesiologists, obstetrician/gynaecologists and other physicians who participate in operative care were eligible to participate.

\section{Ethical considerations}

Ethics approval for the study was obtained from the Stellenbosch University Human Research Ethics Committee (ref. no. N20/08/050_ COVID-19). An explanation of the study was provided in the preface of the online questionnaire and informed consent was implied if the participant completed the questionnaire. All data were anonymous.

\section{Survey}

Participants were asked to score each recommendation on a 5-point Likert scale from 1 (strongly disagree) to 5 (strongly agree). Each participant was asked about local COVID-19 factors in order to create their facility pandemic score. Participants evaluated 10 hypothetical cases representing general, trauma, orthopaedic, neurological, gynaecological, ophthalmological and urological surgical subspecialties based on their pandemic score. In addition, each participant reported whether the case would have been operated on at their hospital facility, given the current pandemic score (supplementary file 1, available at http://samj.org.za/public/ sup/15603-surv.pdf).

\section{Data analysis}

Data were imported from Google drive into Statistical Package for the Social Sciences (SPSS) version 27 (IBM, USA) for statistical analysis. Each recommendation statement was scored from 1 to 5 . Mean scores $\geq 4$ were considered positive support for the statement. For the 10 hypothetical cases, concordance was measured between the COVID-19 surgical risk calculator and the participant decision on the case. Non-concordant responses were further analysed to identify whether the risk calculator was more or less permissive than participants' opinion. The results were analysed using descriptive statistics (frequencies and percentages). Concordance of $>80 \%$, as agreed by the COVID-19 subcommittee a priori, was considered acceptability of the risk calculator. Cronbach's alpha test was used to measure internal consistency for case concordance.

\section{Results}

\section{Literature review}

Thirty documents, including published articles and unpublished sources, were identified: 16 international and 14 SA (Table 1). Recommendations around the prioritisation of surgical care during COVID-19 were put into four categories: local pandemic, hospital 


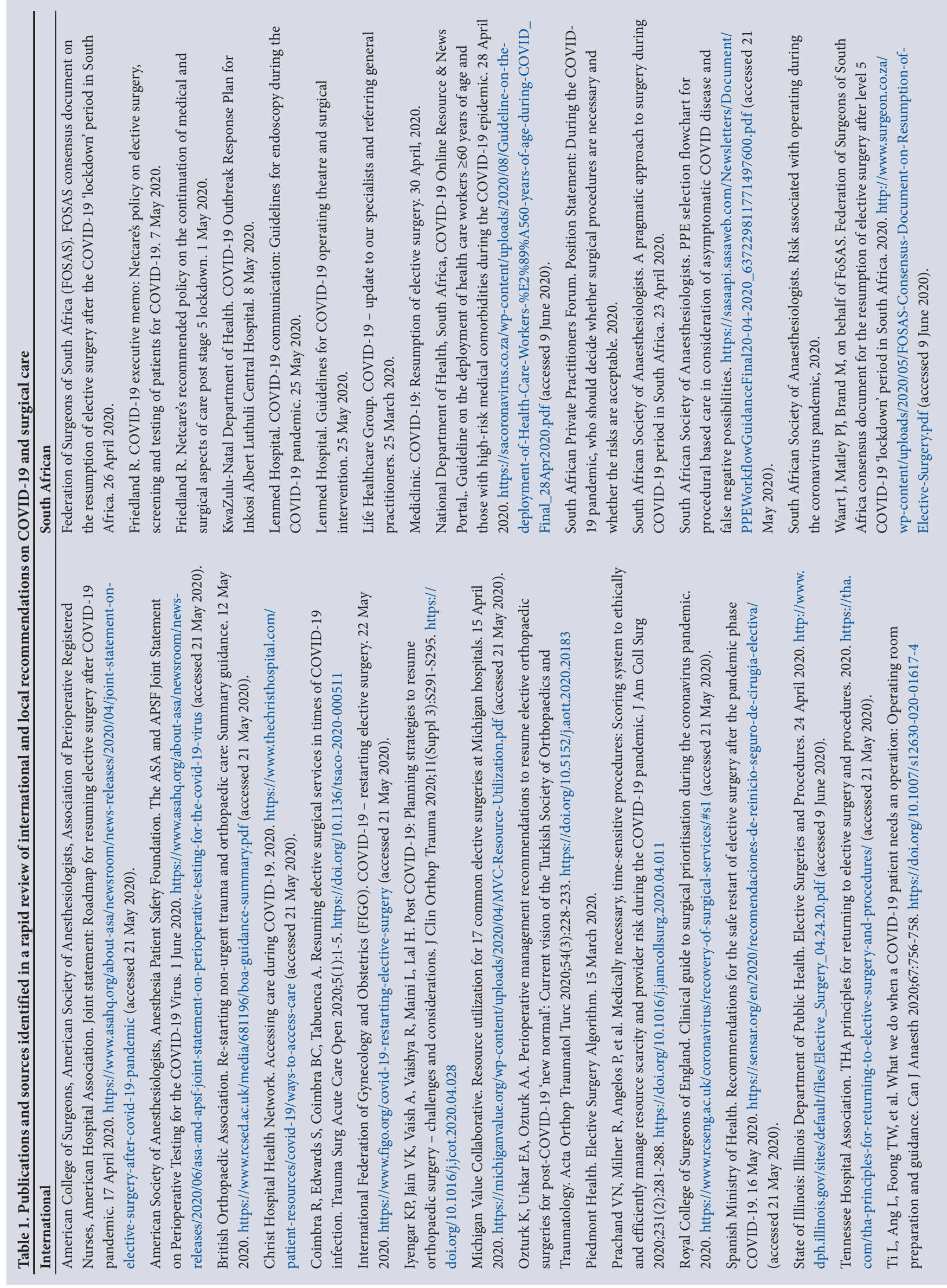


environment, patient, and surgical needs/backlog factors. These were subdivided into COVID-19 epidemiology, COVID-19 screening and testing, hospital bed availability, PPE, staff and equipment availability, dedicated COVID-19 teams, quality of care, prioritisation factors, order of cases, protocol/guidelines, and patient factors. The importance of each of these recommendations was ranked by the task team and incorporated into the national recommendations and risk calculator.

\section{National recommendations}

Six national recommendations were created and are listed in Table 2. These were based on the best evidence in the literature on perioperative mortality of COVID-19-positive patients and infection control measures. A COVID-19 risk calculator was created for the SA context in three parts. The first part, the individual risk assessment score which was based on the MeNTS score, ${ }^{[18]}$ considers disease, procedure, and patient comorbidity factors. Several variables were modified including the effect of delay on the natural history of the disease and some of the procedure categories. In addition, COVID-19 symptoms were removed from the patient factors since preoperative testing was included as a recommendation. The facility pandemic score was created to adjust the final decision for the local COVID-19 context (supplementary file 2, available at http://samj.org.za/public/sup/15603-risk.pdf).

\section{Evaluation of the recommendations and COVID-19 risk calculator}

\section{Participants' demographics}

The demographic characteristics of the study participants are shown in Table 3. A total of 99 participants from eight of the nine SA provinces completed the questionnaire.

\section{Recommendations}

All six recommendations received mean ratings $>4$ on the 5-point Likert scale (Table 2). The recommendations with the highest rankings were the need to assess individual patient risk and health facility readiness (mean 4.7), obtaining consent for risk of COVID-19 infection (mean 4.7) and mandatory COVID-19 testing (mean 4.6).

\section{COVID-19 risk calculator}

The pandemic scores reported by participants ranged from 4 to 16 , with a mean (standard deviation) of 7.1 (3.2). The overall concordance of the COVID-19 surgical risk calculator with current clinical practice was $81 \%$ (Table 4). Further analysis of the $19 \%$ non-concordant responses demonstrated that in circumstances when the risk calculator suggested that surgery be done, the majority (93.6\%) of the healthcare providers would not have carried out surgery in such instances. The Cronbach alpha test for internal consistency was 0.71 .

Table 5 illustrates the feedback of the study participants on the COVID-19 risk calculator. A total of 54 participants (54.5\%) felt that the risk calculator was helpful to either determine individual patient risk or prioritise patients against others. Sixteen participants (16.2\%) said that the risk calculator was not helpful, while 59 (59.6\%) felt it would be a helpful tool for other hospital staff.

\section{Discussion}

There is no panacea for the surgical backlog created by the COVID-19 pandemic. SA has inequitable surgical access, especially in the rural

\begin{tabular}{ll}
$\begin{array}{l}\text { Table 3. Demographic characteristics of the study } \\
\text { participants }(\boldsymbol{N = 9 9 )}\end{array}$ \\
\hline
\end{tabular}

\section{Table 2. Key recommendations on COVID-19 and surgery}

\begin{tabular}{|c|c|c|}
\hline Ranking & Key recommendation & Rating, mean (SD) \\
\hline 1 & $\begin{array}{l}\text { Safe reintroduction of surgery in the presence of community transmission of COVID-19 requires } \\
\text { assessment of individual patient risk as well as health facility readiness }\end{array}$ & $4.7(0.7)$ \\
\hline 2 & $\begin{array}{l}\text { Surgery with coexisting COVID-19 infection poses an increased risk of morbidity and mortality, } \\
\text { and non-emergency surgery should be avoided }\end{array}$ & $4.4(0.9)$ \\
\hline 3 & $\begin{array}{l}\text { Preoperative COVID-19 testing should ideally be performed on all patients scheduled for } \\
\text { non-emergency surgery and surgery postponed if positive (for at least } 10 \text { days after last day } \\
\text { of symptoms or from test if asymptomatic) }\end{array}$ & $4.6(0.8)$ \\
\hline 4 & $\begin{array}{l}\text { Where testing is not available or turnaround time is prohibitively long, patients with } \\
\text { COVID-19 symptoms should be postponed }\end{array}$ & $4.4(0.9)$ \\
\hline 5 & $\begin{array}{l}\text { For patients who have recovered from COVID-19, assessment for COVID- } 19 \text { sequelae is part of } \\
\text { the risk evaluation. Routine repeat PCR testing is not recommended }\end{array}$ & $4.4(0.9)$ \\
\hline 6 & $\begin{array}{l}\text { Risk of concomitant and nosocomial COVID-19 infection should be discussed as part of the } \\
\text { consent process }\end{array}$ & $4.7(0.6)$ \\
\hline
\end{tabular}


Table 4. Concordance of the COVID-19 risk calculator with clinical practice

\begin{tabular}{|c|c|c|c|c|c|}
\hline & \multirow[b]{3}{*}{$n$} & \multicolumn{2}{|c|}{ Clinical decision } & \multicolumn{2}{|c|}{ Non-concordant responses } \\
\hline & & & & Risk calculator: Yes & Risk calculator: No \\
\hline & & Concordant & Not concordant & Clinical practice: No & Clinical practice: Yes \\
\hline Case 1 & 90 & $69(76.7)$ & $21(23.3)$ & $20(95.2)$ & $1(4.8)$ \\
\hline Case 2 & 82 & $68(82.9)$ & $14(17.1)$ & $13(92.9)$ & $1(7.1)$ \\
\hline Case 3 & 83 & $62(74.7)$ & $21(25.3)$ & $19(90.5)$ & $2(9.5)$ \\
\hline Case 4 & 91 & $82(90.1)$ & $9(9.9)$ & $8(88.9)$ & $1(11.1)$ \\
\hline Case 5 & 88 & $72(81.8)$ & $16(18.2)$ & $13(81.3)$ & $3(18.7)$ \\
\hline Case 6 & 80 & $59(73.8)$ & $21(26.3)$ & $20(95.2)$ & $1(4.8)$ \\
\hline Case 7 & 78 & $60(76.9)$ & $18(23.1)$ & $18(100)$ & 0 \\
\hline Case 8 & 77 & $66(85.7)$ & $11(14.3)$ & $11(100)$ & 0 \\
\hline Case 9 & 79 & $76(96.2)$ & $3(3.8)$ & $2(66.7)$ & $1(33.3)$ \\
\hline Case 10 & 79 & $56(70.9)$ & $23(29.1)$ & $23(100)$ & 0 \\
\hline Total & 827 & $670(81.0)$ & $157(19.0)$ & 147 (93.6) & $10(6.4)$ \\
\hline
\end{tabular}

\begin{tabular}{|c|c|}
\hline Variables & $n(\%)$ \\
\hline \multicolumn{2}{|c|}{ Was the COVID-19 surgery risk calculator helpful for you? } \\
\hline Yes* & $54(54.5)$ \\
\hline Maybe/don't know & $29(29.3)$ \\
\hline $\mathrm{No}^{\dagger}$ & $16(16.2)$ \\
\hline \multicolumn{2}{|c|}{ Would this COVID-19 surgery risk calculator help others at your hospital? } \\
\hline Yes & $59(59.6)$ \\
\hline Maybe/don't know & $33(33.3)$ \\
\hline $\mathrm{No}^{\dagger}$ & $7(7.1)$ \\
\hline
\end{tabular}

public sector ${ }^{[19]}$ and between the public and private health systems. The COVID-19 pandemic has exposed inherent weaknesses in the surgical health system throughout the country. Until new infections can be controlled through improved public health measures such as vaccination and non-pharmaceutical interventions, access to surgical care will continue to be limited and variable between facilities and health sectors. Some hospitals will provide a full complement of non-essential and essential operations, while others will only provide the latter. Until now, the process of selecting operations to perform has not been uniform, with decisions about which procedures to cancel, delay or proceed with made on a case-by-case basis. A national framework to guide this decision-making, which has both overarching key recommendations and guidance on how to integrate individual patient risk factors with the rapidly changing intensity of the COVID-19 pandemic, is necessary.

To this end, the national COVID-19 surgical task force has created tools to assist hospital perioperative teams to objectively evaluate risks and benefits of each surgery. Six recommendations around preoperative COVID-19 screening and testing as well as the use of appropriate PPE were created. In addition, a COVID-19 surgical risk calculator was developed and tested, which considers local pandemic severity, as well as patient factors such as anaesthesia risk, disease severity, and SARS-CoV-2 transmission risk during the proposed operation. This risk calculator had a high acceptability and level of concordance with current clinical practice among nearly 100 perioperative practitioners. Looking at discordant cases, practitioners tended to be more conservative than the risk calculator, with the majority cancelling or delaying operations even when the risk calculator recommended proceeding, suggesting that other factors such as fear of poor outcomes or staff and bed shortages could be contributing to their clinical decision-making.

\section{Study limitations}

The limitations of this study are that the COVID-19 pandemic is changing clinical practice rapidly, and the literature review will no longer be up to date at the time of publication. In addition, the snowball sampling frame may have introduced bias, and those who have accepted the risk calculator use may have been more likely to respond, thereby overestimating its acceptance.

\section{Conclusions}

This is the first SA national framework for COVID-19 surgical prioritisation tool, created using the best available evidence and validated by surgical stakeholders. This framework was endorsed by the Ministerial Advisory Committee on COVID-19 on 28 August $2020,{ }^{[20]}$ as well as by the Association of Surgeons of South Africa, but it still needs to be widely implemented. We believe that the COVID19 recommendations and risk calculator are useful tools for the SA context, which may help perioperative teams provide equitable, ethical and personalised patient care.

\section{Declaration. None.}

Acknowledgements. The authors acknowledge all the frontline workers of the COVID-19 pandemic in South Africa.

Author contributions. KMC and MS conceived the study design and wrote the first draft. LC, BMB and MM created the first draft of the risk calculator. JM designed the data collection tool. EOO analysed the data. 
KMC, TCH, SM, PDG, HG and LC performed the literature review. All authors contributed to manuscript revisions and approved the final version. Funding. None.

Conflicts of interest. None.

1. World Health Organization. WHO Coronavirus Disease (COVID-19) Dashboard 2021. https:// covid19.who.int/table (accessed 1 February 2021)

2. Spinelli A, Pellino G. COVID-19 pandemic: Perspectives on an unfolding crisis. Br J Surg 2020;107(7):785-787. https://doi.org/10.1002/bjs.11627

3. American College of Surgeons. COVID-19: Recommendations for management of elective surgical procedures. 13 March 2020. https://www.facs.org/covid-19/clinical-guidance/elective-surgery (accessed 21 May 2020)

4. COVIDSurg Collaborative. Elective surgery cancellations due to the COVID-19 pandemic: Global predictive modelling to inform surgical recovery plans. Br J Surg 2020;107(11):1440-1449. https://doi. org $/ 10.1002 / \mathrm{bjs} .11746$

5. IOL News. Coronavirus: Gauteng Health announces additional measures to deal with Covid-19. 22 March 2020. https://www.iol.co.za/news/south-africa/gauteng/coronavirus-gauteng-healthannounces-additional-measures-to-deal-with-covid-19-45364314 (accessed 21 December 2020).

6. Medical Brief. SA hospital groups pinched as elective surgeries end and occupancies plummet. $20 \mathrm{May}$ 2020. https://www.medicalbrief.co.za/archives/sa-hospital-groups-pinched-as-elective-surgeries-endand-occupancies-plummet/ (accessed 21 December 2020).

7. Rosen K. Elective surgeries may be suspended in Manitoba as hospitalizations climb. Winnipeg News, 28 October 2020. https://winnipeg.ctvnews.ca/elective-surgeries-may-be-suspended-in-manitoba-ashospitalizations-climb-1.5164103 (accessed 21 December 2020).

8. Chu K, Smith M, Steyn E, Goldberg P, Bougard H, Buccimazza I. Changes in surgical practice in 85 South African hospitals during COVID-19 hard lockdown. S Afr Med J 2020;110(9):916-919. https:// doi.org/10.7196/SAMJ.2020.v110i9.15014

9. Hubner M, Zingg T, Martin D, Eckert P, Demartines N. Surgery for non-Covid-19 patients during the pandemic. PLoS ONE 2020;15(10):e0241331. https://doi.org/10.1371/journal.pone.0241331

10. Jain A, Jain P, Aggarwal S. SARS-CoV-2 impact on elective orthopaedic surgery: Implications for postpandemic recovery. J Bone Joint Surg Am 2020;102(13):e68. https://doi.org/10.2106/JBJS.20.00602
11. Moustakis J, Piperidis AA, Ogunrombi AB. The effect of COVID-19 on essential surgical admissions in South Africa: A retrospective observational analysis of admissions before and during lockdown at in South Africa: A retrospective observational analysis of admissions before and during lockdown at v110i9.15025

12. Patriti A, Baiocchi GL, Catena F, Marini P, Catarci M, FACS on behalf of the Associazione Chirurghi Orpedalieri Italini (ACOI). En Ospedalieri Italiani (ACO). Emergency general surgery in Italy during the COVID-19 outbreak: First survey from the real life. World J Ferg Surg 2020;15(1):36-43. https//doi 0 rg/10.1186/s13017-020-

13. Wang J, Vahid S, Eberg M, et al. Clearing the surgical backlog caused by COVID-19 in Ontario: A time series modelling study. Can Med Assoc J 2020;192(44):E1347-E1356. https://doi.org/10.1503/ cmaj.201521

14. Meara JG, Leather AJM, Hagander L, et al. Global Surgery 2030: Evidence and solutions for achieving health, welfare, and economic development. Lancet 2015;386(9993):569-624. https://doi.org/10.1016/ S0140-6736(15)60160-X

15. Biccard B, Cairncross L. Over $70 \%$ of surgeries in SA will be cancelled or postponed due to Covid-19 - how will we catch up? Daily Maverick, 31 May 2020. https://www.dailymaverick.co.za/article/2020-0531-over-70-of-surgeries-in-sa-will-be-cancelled-or-postponed-due-to-covid-19-how-will-we-catch-up/ (accessed 21 December 2020).

16. American College of Surgeons, American Society of Anesthesiologists, Association of Perioperative Registered Nurses, American Hospital Association. Joint statement: Roadmap for resuming elective surgery after COVID-19 pandemic. 17 April 2020. https://www.asahq.org/about-asa/newsroom/newsreleases/2020/04/joint-statement-on-elective-surgery-after-covid-19-pandemic (accessed 21 May 2020). 17. Royal College of Surgeons of England. Clinical guide to surgical prioritisation during the coronavirus pandemic. 2020. https://www.rcseng.ac.uk/coronavirus/recovery-of-surgical-services/\#s1 (accessed 21 May 2020).

18. Prachand VN, Milner R, Angelos P, et al. Medically necessary, time-sensitive procedures: Scoring system to ethically and efficiently manage resource scarcity and provider risk during the COVID-19 pandemic. J Am Coll Surg 2020;231(2):281-288. https://doi.org/10.1016/j.jamcollsurg.2020.04.011

19. Dell AJ, Kahn D, Klopper J. Surgical resources in South Africa: An analysis of the inequalities between the public and private sector. S Afr J Surg 2018;56(2):16-20. https://doi.org/10.17159/2078-5151/2018/ v56n2a2397

20. National Department of Health, South Africa, COVID-19 Online Resource \& News Portal. COVIDsurgery recommendations and risk calculator. 28 August 2020. https://sacoronavirus.co.za/2020/08/28/ covid-surgery-recommendations-and-risk-calculator/ (accessed 21 December 2020).

Accepted 5 March 2021. 